\title{
On numerical simulation of stirring in stir casting process
}

\author{
Vishnu Prasad K ${ }^{1}$ and K. R. Jayadevan²* \\ Department of Mechanical Engineering \\ ${ }^{1}$ Government Engineering College, Thrissur, Kerala, 680009, India. \\ ${ }^{2}$ Government Engineering College, Palakkad, Kerala, 678633, India. \\ *Email: jayadevankr@gecskp.ac.in
}

Stir casting is the cheapest, most flexible and simplest of all AMC manufacturing techniques in industrial use [1]. In Stir casting process, the properties achieved by the final cast strongly depend upon the homogeneity of the particle distribution during the solidification of the melt. Hence, it is important to study the effect of stirring parameters on the uniformity of particle distribution.

Recently, Naher et. al. [2] conducted analogous experiments using water model to study the effect of stirring. In the reported numerical studies [3, 4], velocity gradient is used for quantifying the uniformity of particle distribution. However, shear rate is the key factor for dispersion of powder in molten aluminium where viscous forces are dominant, and hence, it is employed in this work.

Single phase computer simulations of the process replicating the published experimental work [2] are carried out using ANSYS-Fluent. Analyses were performed with different stirring speeds, number of blades and blade angles. The rate of shear deformation tensor is found using by using eq. 1 .

$S_{i j}=\left(\frac{1}{2}\left(\partial_{i} u_{j}+\partial_{j} u_{i}\right)\right)-\frac{1}{3} \sum_{k}\left(\partial_{k} u_{k}\right) \delta_{i j}$

The magnitude of shear rate is the resultant of inner product of rate of shear deformation tensor. This value is then found for each cell in the mesh. The volume average of shear rate of the entire melt domain is computed. An increase in the shear rate indicates a corresponding increase in mixing efficiency which in-turn leads to decrease in time required for uniform particle distribution.

A typical result for the case of 3 bladed stirrer with a blade angle of $90^{\circ}$, showing the variation of shear rate at the instant of uniform particle distribution with stirring speed are presented in Figure 1. For comparison, inverse of the experimental time for uniform particle distribution [2] are also shown in the same figure. The simulated results show that the stirring speed strongly enhances the shear rate, and hence the particle distribution, which qualitatively agrees with the experimental results. Similar agreement was also noticed in all the other cases. However, unlike the variation with stirring speed, the effect of blade angle and number of blades on particle distribution was found to be less pronounced.

Pearson correlation coefficient between volume average of shear rate and inverse of time for uniform particle dispersion was found for each case. The values of the coefficient obtained were in the range of $0.8-0.95$, which show good correlation between the data.

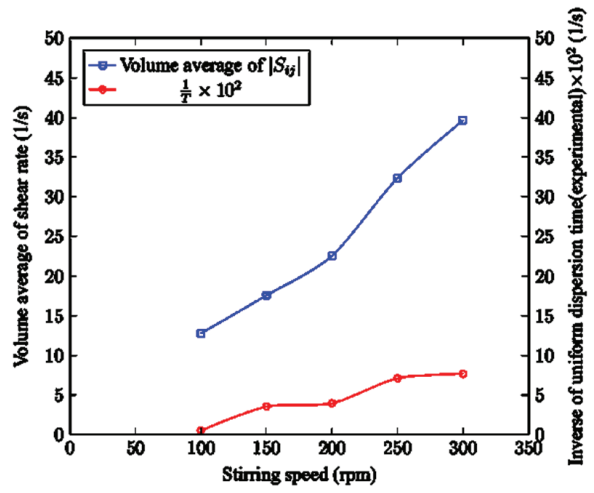

Figure 1: Variation of computed shear rate with stirring speed for a 3 bladed stirrer with blade angle of $90^{\circ}$. Experimental results [2] (Inverse of time for uniform particle distribution) is also shown

Further, an equation to predict the dispersion time from shear rate was obtained using regression analysis. This enables the researchers to estimate the time for uniform particle dispersion in the process from simple single phase simulation data.

\section{References}

1. Mirle K Surappa, Sadhana, 28 (2003) 319-334.

2. Sumsun Naher, Dermot Brabazon, Lisa Looney, J. Mater. Process. Tech., 143-144 (2003) 567-571.

3. Sumsun Naher, Dermot Brabazon, Lisa Looney, Composites: Part A, 38 (2007) 719-729.

4. Hai Su, Wenli Gao, Hui Zhang, Hongbo Liu, Jian Lu, Zheng Lu, J. Manuf. Sci. Eng., 132 (2010) 061007 (1-7). 